\title{
Prevalence of a Second Root and Canal in Mandibular and Maxillary Canines in a Saudi Arabian Population: A Cone-beam Computed Tomography Study
}

\author{
Mohammed Mashyakhy
}

\begin{abstract}
Aim: This study evaluated the prevalence of a second root and canal, gender differences, and bilateral symmetry of mandibular and maxillary permanent canines in a Saudi Arabian population using a cone-beam computed tomography (CBCT).

Materials and methods: The first step was to evaluate CBCT images of 208 patients with 794 canines (410 mandibular and 384 maxillary canines). The second step was to identify the number of roots, number of canals, and canal configurations based on Vertucci's classification to investigate the prevalence of a second root and a second canal, gender differences, and bilateral symmetry. A $Z$ test evaluated differences in the independent proportions; a Chi-square test determined differences between genders and Cohen's Kappa test assessed bilateral symmetry. Results: The prevalence of two roots and two canals in mandibular canines was $2.7 \%$ and $9.3 \%$, respectively, and Vertucci's type III appeared in $6.1 \%$, and Vertucci's type V appeared in 3.2\%. In maxillary canines, there were no two-rooted teeth, and only $1 \%$ of two canals were present with Vertucci's type III. There were no statistically significant differences between genders in both mandibular and maxillary canines. High bilateral symmetries of roots and canals appeared in mandibular canines ( $95.5 \%$ and $91.1 \%$, respectively) and maxillary canines (100\% and $98.9 \%$, respectively).

Conclusion: One root, one canal, and Vertucci's type I are the primary and typical characteristics of mandibular and maxillary canines. The presence of a second root in mandibular canines is small, although the presence of two canals is not unusual. Conversely, it is rare to find a second root and canal in maxillary canines.

Clinical significance: Mandibular canines showed bilateral asymmetry (8.9\%) in the number of canals between the right and left sides, which could be of clinical importance when planning root canal treatment (RCT) on contralateral canines.

Keywords: Canines, CBCT, Root canal morphology, Saudi population.

The Journal of Contemporary Dental Practice (2019): 10.5005/jp-journals-10024-2595
\end{abstract}

\section{INTRODUCTION}

Increasing knowledge of rootcanal morphology is essential for proper root canal treatment (RCT). Anterior teeth in the maxillary jaw mostly havea simpleand straightforward morphologythatgenerallypresents with one root and one canal. Mandibular anterior teeth have a more complex anatomy and the presence of two canals is common. ${ }^{1-5}$ Mandibular permanent canines typically have a single root with one root canal. ${ }^{4,5}$ Many studies on mandibular canines from different populations reported the presence of up to two roots and two canals with Vertucci's types I-IV canal configurations. ${ }^{6-12}$ Two-rooted mandibular canines have reached $5 \%{ }^{5,7}$, while single-rooted canines with two root canals were reported to be approximately $15 \% .^{1,4,13}$

In a Saudi Arabian population, only one recent in vivo cone-beam computed tomography (CBCT) study evaluated the anatomy of permanent mandibular canines. ${ }^{14}$ In this study, the authors evaluated 454 mandibular canine teeth and found that a single-rooted canine with Vertucci's type I classification was the most prevalent type (95.4\%). Two-rooted mandibular canines comprised $0.2 \%$ and two canals were present in $4.6 \%$ of the cases. The remaining studies conducted with the same population were case reports of anatomical variations. ${ }^{15-18}$

Conversely, few studies have evaluated the morphology of permanent maxillary canines. Maxillary canines usually present with a single root and a single canal; the presence of two roots or canals is rare. ${ }^{2,19}$ In recent in vivo $\mathrm{CBCT}$ studies among different populations, Martins et al. ${ }^{3}$ reported that out of 962 teeth, maxillary canines exhibited one root (100\%) and one canal (98.6\%) and the presence
Department of Restorative Dental Sciences, College of Dentistry, Jazan University, Kingdom of Saudi Arabia

Corresponding Author: Mohammed Mashyakhy, Department of Restorative Dental Sciences, College of Dentistry, Jazan University, Kingdom of Saudi Arabia, Phone: +966 557224154, e-mail: dr.mashyakhy@gmail.com How to cite this article: Mashyakhy M. Prevalence of a Second Root and Canal in Mandibular and Maxillary Canines in a Saudi Arabian Population: A Cone-beam Computed Tomography Study. J Contemp Dent Pract 2019;20(7):773-777.

Source of support: Nil

Conflict of interest: None

of two canals was $1.4 \%$. Pan et al. found all maxillary canines having one root and one canal Vertucci's type I. ${ }^{20}$

An extensive literature search revealed no studies that reported anatomy of permanent maxillary canines and only one study evaluated permanent mandibular canines in a Saudi Arabian population. Therefore, the present study evaluates (a) the prevalence of a second root and canal in mandibular and maxillary canines, (b) gender differences, and (c) bilateral symmetry of canines morphology in a Saudi Arabian population using in vivo CBCT.

\section{Materials and Methods}

CBCT images were retrieved from the database of College of Dentistry, Jazan University, Jazan, Saudi Arabia from 2016 to 2018, following receipt of the Institute's ethical research approval. This 
study included mandibular and maxillary permanent canines with fully developed roots and closed apices. This study excluded root canal-treated, calcification, resorption, or distorted CBCT images. The CBCT machine used in this in vivo retrospective study was a 3D Accuitomo 170 (MORITA, Japan) with the scanning parameters: FOV 170-120 mm, $90 \mathrm{kV}, 5-8 \mathrm{~mA}, 17.5$ seconds exposure time, and 0.250 $\mathrm{mm}$ voxel size. All CBCT images were processed and reconstructed using Morita's i-Dixel 3D imaging software, and acquired serial axial, coronal, and sagittal sections to evaluate external and internal morphology of maxillary molars. The same endodontist assessed the CBCT images twice, with a 5-week interval in between each assessment, and evaluated the following parameters:

- The number of roots, number of canals, and canals' configurations according to Vertucci's classification (Fig. 1) in mandibular and maxillary permanent canines.

- The prevalence of a second root and a second canal.

- Differences between genders.

- Bilateral symmetry of the number of roots, number of canals, and canals configurations.

\section{Statistical Analysis}

The researcher entered the collected data into the Statistical Package of Social Sciences software program for Windows (SPSS V25; IBM, Chicago, IL), followed by coding and analysis. The results report frequencies and percentages with a $95 \%$ confidence interval $(\mathrm{Cl})$. The $Z$ test determined whether differences existed in the independent groups. A Chi-square test assessed differences between males and females as well as left and right locations, and Cohen's Kappa test determined the presence of bilateral symmetry and assessed intrarater reliability. The level of significance for all statistical tests was set at $p<0.05$.

\section{Results}

For reliability, one observer (the author) evaluated $30 \%$ of the total sample twice with an interval period of 5 weeks in between

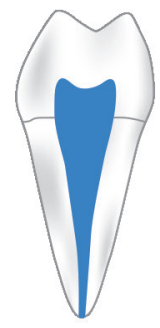

Type I

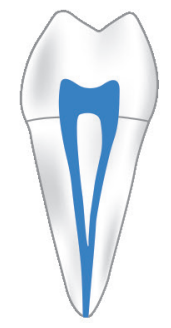

Type II

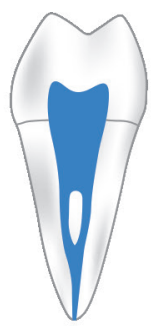

Type III

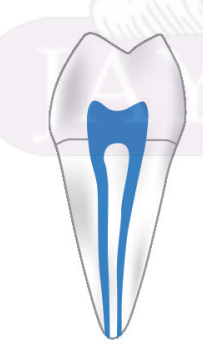

Type IV

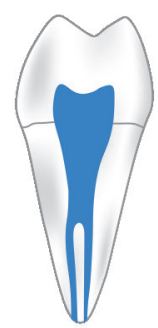

Type V

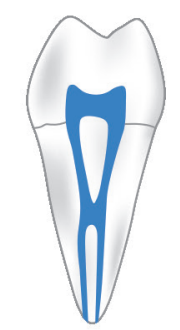

Type VI

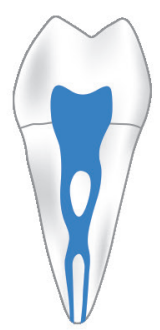

Type VII

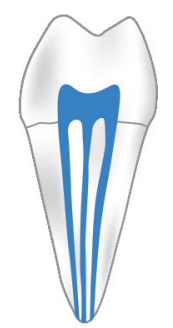

Type VIII each evaluation. Results of Cohen's Kappa test revealed an almost perfect agreement between observations with a value of $85.8 \%$ and asymptotic standard error of $\pm 9.8 \%$.

\section{Study Sample}

A total of 794 mandibular and maxillary canines (410 mandibular and 384 maxillary canines) were evaluated. These teeth were examined from the CBCT radiographs of 208 subjects including 100 (48\%) males and 108 (52\%) females and whose ages ranged from 17 to 62 years. Mean age $28.95 \pm 9.80$ years (median $=26$ years).

\section{Prevalence of Second Root and Canal}

Of the 410 mandibular canines, 339 (97.3\%) teeth had one root and $11(2.7 \%)$ teeth had two roots. Additionally, 372 (90.7\%) of the teeth had one canal and 38 (9.3\%) teeth had two canals (Fig. 2). Vertucci's type I was present in $372(90.7 \%)$ teeth, while 25 (6.1\%) teeth had Vertucci's type III and 13 (3.2\%) teeth had Vertucci's type V (Table 1).

Although 384 maxillary canines showed one root (100\%), 380 (99.0\%) had one canal, and 4 (1.0\%) teeth had two canals (Fig. 3). Similarly, 380 (99.0\%) teeth had Vertucci's type I and 4 (1.0\%) teeth had Vertucci's type III (Table 1).

\section{Gender Differences}

Among the mandibular canines, there were no statistically significant differences between genders regarding the number of roots ( $p=0.064)$ and canals $(p=0.088)$. Regarding canal configurations, there was a statistically significant difference between genders but it was near the cutoff point ( $p=0.049)$ (Table 2). Among the 197 mandibular canines in males, 195 (99.0\%) had one root and $2(1.0 \%)$ had two roots. Among 213 mandibular canines in females, 204 (95.8\%) teeth had one root and 9 (4.2\%) had two roots. In males, 184 (93.4\%) mandibular canines had one canal; in females, 188 (88.3\%) mandibular canines had one canal. Vertucci's type I appeared more frequently in males than it did in females (93.4\% compared to $88.3 \%$ ), while Vertucci's types III and $\checkmark$ were more frequent in females than in males.

Among the maxillary canines, males and females had one root on both sides and no significant differences emerged regarding the number of canals $(p=0.052)$ and canal configurations $(p=0.052)$ (Table 2). There were $200(52 \%)$ teeth in females and $180(48 \%)$ teeth in males. All teeth $(100 \%)$ in both genders had one root. All teeth (100\%) in females had one canal, while, in males, 180 (97.8\%) teeth had one canal and $4(2.2 \%)$ teeth had 2 canals. Similarly, all teeth (100\%) in females had Vertucci's type I, while 180 (97.8\%) teeth in males had Vertucci's type I and 4 (2.2\%) teeth had Vertucci's type III.

Table 1: Frequency of number of roots, number of canals, and Vertucci type

\begin{tabular}{cll}
\hline & Mandibular canines & Maxillary canines \\
\hline Number of roots & & \\
1 root & $399(97.3)$ & $384(100.0)$ \\
2 roots & $11(2.7)$ & - \\
Number of canals & & $380(99.0)$ \\
1 canal & $372(90.7)$ & $4(1.0)$ \\
2 canals & $38(9.3)$ & \\
Vertucci types & & $380(99.0)$ \\
Type I & $372(90.7)$ & $4(1.0)$ \\
Type III & $25(6.1)$ & - \\
Type V & $13(3.2)$ & \\
\hline
\end{tabular}

Fig. 1: Vertucci's classification of the root canal system 


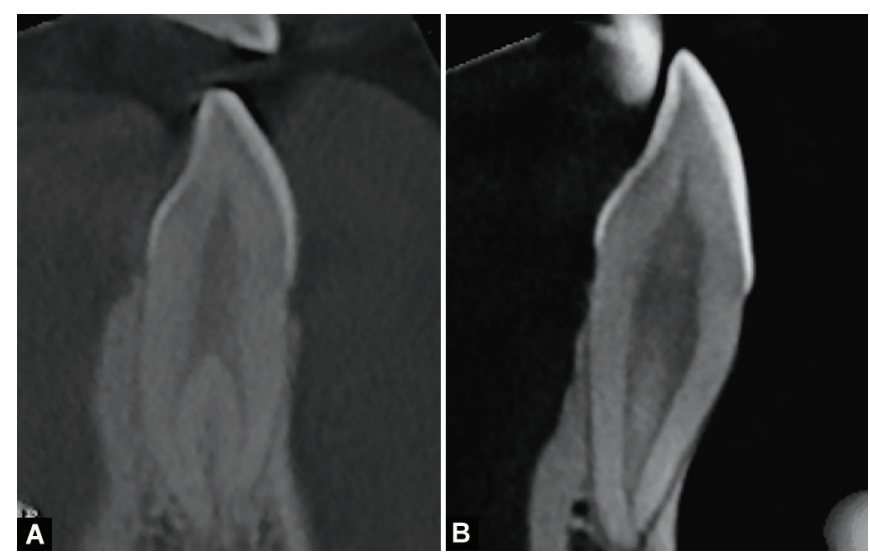

Figs $2 \mathrm{~A}$ and $\mathrm{B}$ : Mandibular canine: $\mathrm{A}$ ) CBCT coronal section shows two roots; (B) $C B C T$ coronal section shows one root with two canals
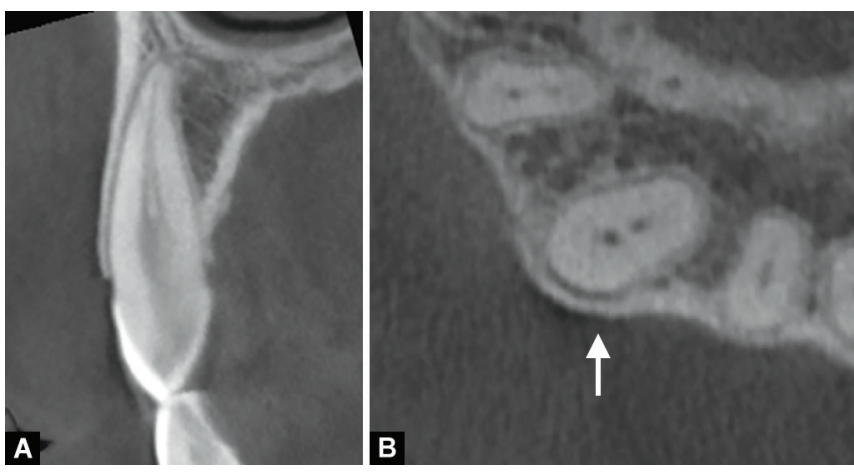

Figs $3 A$ and B: Maxillary canine with two canals: (A) CBCT coronal section shows two canals; (B) CBCT axial section "arrow" shows two canals

Table 2: Comparison between males and females in relation to the study variables

\begin{tabular}{|c|c|c|c|c|c|c|c|c|}
\hline & \multicolumn{4}{|c|}{ Mandibular canines } & \multicolumn{4}{|c|}{ Maxillary canines } \\
\hline & Male & Female & Total & $p$ & Male & Female & Total & $p$ \\
\hline \multicolumn{9}{|c|}{ Number of roots } \\
\hline 1 root & $195(99.0)$ & $204(95.8)$ & $399(97.3)$ & 0.064 & $184(100.0)$ & $200(100.0)$ & $384(100.0)$ & NC \\
\hline 2 roots & $2(1.0)$ & $9(4.2)$ & $11(2.7)$ & & & & & \\
\hline Total & $197(48.0)$ & $213(52.0)$ & $410(100.0)$ & & $184(0.48)$ & $200(0.52)$ & $384(100.0)$ & \\
\hline \multicolumn{9}{|c|}{ Number of canals } \\
\hline 1 canal & $184(93.4)$ & $188(88.3)$ & $372(90.7)$ & 0.088 & $180(97.8)$ & $200(100.0)$ & $380(99.0)$ & 0.05 \\
\hline 2 canals & $13(6.6)$ & $25(11.7)$ & $38(9.3)$ & & $4(2.2)$ & $0(0.0)$ & $4(1.0)$ & \\
\hline Total & $197(48.0)$ & $213(52.0)$ & $410(100.0)$ & & $184(0.48)$ & $200(0.52)$ & $384(100.0)$ & \\
\hline \multicolumn{9}{|l|}{ Vertucci types } \\
\hline Type I & $184(93.4)$ & $188(88.3)$ & $372(90.7)$ & $0.049^{*}$ & $180(97.8)$ & $200(100.0)$ & $380(99.0)$ & 0.05 \\
\hline Type III & $11(5.6)$ & $14(6.6)$ & $25(6.1)$ & & $4(2.2)$ & $0(0.0)$ & $4(1.0)$ & \\
\hline Type V & $2(1.0)$ & $11(5.2)$ & $13(3.2)$ & & & & & \\
\hline Total & $197(48.0)$ & $213(52.0)$ & $410(100.0)$ & & $184(0.48)$ & $200(0.52)$ & $384(100.0)$ & \\
\hline
\end{tabular}

The Chi-squared test was used; NC, not computed; *significant at $p<0.05$

\section{Bilateral Symmetry}

Among the mandibular canines, 202 of 208 participants (97.1\%) had both right and left mandibular canines. The bilateral symmetry for the number of roots was $95.5 \%(p=0.023)$; the majority of participants ( $n=192 ; 95.1 \%$ ) had one root on both sides and one participant $(0.5 \%)$ had two roots on both sides. Bilateral symmetry for the number of canals was $91.1 \%(p<0.001) ; 86.1 \%(n=174)$ of participants had one canal on both sides and only $4.9 \%(n=10)$ had two canals on both sides. Regarding canal configurations, the total bilateral symmetry was $90.1 \%(p<0.001) ; 86.1 \%(n=174)$ of the participants had Vertucci's type I on both sides, 3.5\% $(n=7)$ of participants had Vertucci's type III on both sides, and only one participant (0.5\%) had Vertucci's type V on both sides (Table 3).

Regarding the maxillary canines, as shown in Table 4, 183 (88.0\% of the total sample) with maxillary canines on both sides had one root in both the left and right sides (100\% bilateral symmetry). However, bilateral symmetry for the number of canals was $98.9 \%$ ( $p<0.001) ; 180$ (98.4\%) participants had one canal on both sides and one participant (0.5\%) had two canals on both sides. Similarly,
Table 3: Bilateral symmetry of the study variables among participants for mandibular canines

\begin{tabular}{|c|c|c|c|c|c|}
\hline & & Left roots\# & & & $p$ \\
\hline & & 1 root & 2 roots & & \\
\hline \multirow[t]{4}{*}{ Right roots\# } & 1 root & $192(95.1)$ & $4(1.9)$ & & $0.023^{*}$ \\
\hline & 2 roots & $5(2.5)$ & $1(0.5)$ & & \\
\hline & & Left canals\# & & & $p$ \\
\hline & & 1 canal & 2 canals & & \\
\hline \multirow[t]{4}{*}{ Right canals\# } & 1 canal & $174(86.1)$ & $9(4.5)$ & & $<0.001^{*}$ \\
\hline & 2 canals & $9(4.5)$ & $10(4.9)$ & & \\
\hline & & Left Vertucci & & & $p$ \\
\hline & Type I & Type III & Type V & & \\
\hline \multirow[t]{3}{*}{ Right Vertucci } & Type I & $174(86.1)$ & $4(1.9)$ & $5(2.5)$ & $<0.001^{*}$ \\
\hline & Type III & $5(2.5)$ & $7(3.5)$ & $0(0.0)$ & \\
\hline & Type V & $4(1.9)$ & $2(1.1)$ & $1(0.5)$ & \\
\hline
\end{tabular}

Cohen's Kappa test was used; *significant at $p<0.05$

Bold values refer to the symmetry between the variables 
Table 4: Bilateral symmetry of the study variables among participants for maxillary canines

\begin{tabular}{|c|c|c|c|c|}
\hline & & Left roots\# & & $p$ \\
\hline & & 1 root & & \\
\hline Right roots\# & 1 root & $183(100.0)$ & & NC \\
\hline & & Left canals\# & & $p$ \\
\hline & & 2 canals & & \\
\hline Right canals\# & 1 canal & $180(98.4)$ & $2(1.1)$ & $<0.001^{*}$ \\
\hline & 2 canals & $0(0.0)$ & $1(0.5)$ & \\
\hline & & Left Vertucci & & $p$ \\
\hline & & Type I & Type III & \\
\hline Right Vertucci & Type I & $180(98.4)$ & $2(1.1)$ & $<0.001 *$ \\
\hline & Type III & $0(0.0)$ & $1(0.5)$ & \\
\hline
\end{tabular}

Cohen's Kappa test was used; NC, not computed; * significant at $p<0.05$

the bilateral symmetry for Vertucci types was $98.9 \%(p<0.001)$. Of 183 participants, 180 (98.4\%) had Vertucci's type I on both sides and $1(0.5 \%)$ participant had Vertucci's type III on both sides.

\section{Discussion}

Researchers have investigated root canal anatomy using different in vitro and in vivo techniques and, recently, the use of in vivo $C B C T$ has become paramount to obtaining proper details of the root canal morphology during the dental visit. ${ }^{21,22} \mathrm{CBCT}$ is a $3 \mathrm{D}$ technology with low radiation dose, ${ }^{22}$ which allows for the examination of a relatively large sample size safely and noninvasively to achieve a reliable prevalence. ${ }^{3,23}$

The present in vivo $\mathrm{CBCT}$ study investigated the prevalence of a second root and canal of mandibular and maxillary canines. The prevalence of two roots and two canals in mandibular canines was $2.7 \%$ and $9.3 \%$, respectively. In teeth with two canals, internal configurations were Vertucci's type III in $6.1 \%$ and type $\mathrm{V}$ in $3.2 \%$. Our results revealed a higher prevalence than a finding in a recently published study in a Saudi subpopulation, ${ }^{14}$ where they reported $0.2 \%$ and $4.6 \%$ for roots and canals, respectively. The authors of that study found Vertucci's canal configuration types I-IV in teeth with two canals. In the same study, Aldahman et al. ${ }^{14}$ evaluated 454 mandibular canines using in vivo CBCT in a central region of Saudi Arabia (Riyadh city). The differences and inconsistencies of their results and ours might be explained by the regional differences where the study samples were obtained, since our sample came from the southern region of Saudi Arabia (Jazan city). Additionally, the small sample size in both studies might be another reason for the differences, suggesting the need for a larger sample size from different regions of the country to extract outcomes that are more reliable.

Other studies from different populations found two-rooted mandibular canines to be in a range of $0-11.54 \%{ }^{6-12}$ and two canals in a range of $0-15.1 \%$. 4,6,7,9-13,20,24-31

In maxillary canines from the present study, there were no two-rooted teeth and only $1 \%$ two canals were present. Vertucci's canal configuration type III appeared in teeth with two canals. Our results are consistent with those of an in vivo CBCT study from a Portuguese population in which all teeth showed only one root and two canals present in only $1.4 \%$ of 962 maxillary teeth with Vertucci's type II, III, or IV in canines with two canals. A 2019 in vivo $\mathrm{CBCT}$ study in Malaysian population reported only one root and one Vertucci's type I canal classification in 404 maxillary canines. ${ }^{20}$ Our study is the first in a Saudi population that evaluated maxillary canines and it seems that the presence of two roots or canals is rare in this population as well.

Concerning gender, our study showed no significant differences in the number of roots and canals in males and female in both mandibular and maxillary canines. However, there was a statistically significant difference (near to the cutoff point $p=0.049$ ) between genders regarding canal configurations, where Vertucci's types III and $\mathrm{V}$ were more prevalent in females than in males, and type I was more prevalent in males than in females. This finding is in partial agreement with a study of the same population on mandibular canines in which there was no significant difference in the number of roots between genders, although the presence of two canals was significantly higher in males. ${ }^{14}$ This study is in contrast with our findings regarding canal configurations, where types II, III, and IV canal configurations were more prevalent in male patients and type I in female patients with a statistically significant difference between the two.

Pan et al. ${ }^{20}$ reported results similar to ours, noting no differences between genders in regard to the number of roots and canals. Soleymani et al. ${ }^{12}$ studied an Iranian population that showed that males have a higher prevalence of two roots and double canals in mandibular canines; in contrast, a study of a Portuguese population by Martins et al. ${ }^{32}$ revealed that women had significantly more roots and root canals than men.

In maxillary canines, our result is consistent with other studies from different populations ${ }^{20,32}$ in that studies showed no differences between males and females in regard to the number of roots and canals.

The present study also investigated bilateral symmetry and found high bilateral symmetry $95.5 \%, 91.1 \%$, and $90.1 \%$ for the number of roots, number of canals, and canal configurations, respectively, in mandibular canines. These findings are consistent with two studies carried out in Saudi ${ }^{14}$ and $\operatorname{Iranian}^{12}$ populations, which reported a high probability of bilateral symmetry of $97 . \%$ and $95.4 \%$ in the number of roots and canal configuration, respectively.

Maxillary canines showed higher similarities in the left and right sides, with $100 \%$ for the number of roots, $98.9 \%$ for the number of canals, and $98.9 \%$ for canal configurations. There were no studies to compare bilateral symmetry of maxillary canines; however, it was not of clinical significance. In contrast, the asymmetry in the number of canals (8.9\%) and canal configurations (9.9\%) of mandibular canines could be of clinical significance when a practitioner treats opposing canines in the same patient. The researchers of a 2019 systematic review of 102,610 teeth using in vivo CBCT from 28 countries concluded that some groups of teeth had a similar morphology among different populations, such as maxillary anterior teeth, while other groups of teeth showed a range of diversity. ${ }^{33}$ Therefore, the results of this anatomical study on mandibular and maxillary canines were within the global range and did not show notable diversity.

\section{Conclusion}

Within the limitations of the present study, mandibular canines showed a higher prevalence of two roots and canals $(2.7 \%$ and $9.3 \%$, respectively) compared to maxillary canines $(0 \%$ and $1 \%$, respectively). Gender had no association with the number of roots and canals in mandibular and maxillary canines. Mandibular canines showed higher asymmetries in the number of roots (4.5\%) and in the number of canals (8.9\%) when compared to maxillary canines 
( $0 \%$ and $1.1 \%$, respectively). Therefore, this finding could be of clinical importance in RCT related to mandibular canines.

\section{References}

1. Vertucci FJ. Root canal anatomy of the human permanent teeth. Oral Surg Oral Med Oral Pathol 1984 Nov;58(5):589-599. DOI: 10.1016/0030-4220(84)90085-9.

2. Vertucci FJ. Root canal morphology and its relationship to endodontic procedures. Endod Top 2005;10(5):3-29. DOI: 10.1111/j.16011546.2005.00129.x.

3. Martins JNR. Root and root canal morphology of the permanent dentition in a Caucasian population: a cone-beam computed tomography study. 2017;50(11):1013-1026. DOI: 10.1111/iej.12724.

4. Pineda F, Kuttler Y. Mesiodistal and buccolingual roentgenographic investigation of 7,275 root canals. Oral Surg Oral Med Oral Pathol 1972 Jan;33(1):101-110. DOI: 10.1016/0030-4220(72)90214-9.

5. Ouellet R. Mandibular permanent cuspids with two roots. J Can Dent Assoc 1995 Feb;61(2):159-161.

6. Aminsobhani $M$, Sadegh $M$, et al. Evaluation of the root and canal morphology of mandibular permanent anterior teeth in an Iranian population by cone-beam computed tomography. J Dent (Tehran) 2013 May;10(4):358-366.

7. Pecora JD, Sousa Neto MD, et al. Internal anatomy, direction and number of roots and size of human mandibular canines. Braz Dent $J$ 1993;4(1):53-57.

8. Zhao Y, Dong Y, et al. Cone-beam computed tomography analysis of root canal configuration of 4674 mandibular anterior teeth. Beijing Da Xue Xue Bao [Internet]. 2014 Feb;46(1):95-99. Available from: http:// europepmc.org/abstract/MED/24535357.

9. Mirzaie M, Zaban PT, et al. Cone-beam Computed Tomography Study of Root Canals in a Hamadani Population in Iran. Avicenna J Dent Res 2012;4:93-99.

10. Singh S, Pawar M. Root and canal morphology of mandibular incisors and canines in South Asian Indian population by canal staining and tooth clearing technique. Endodontology 2016;28:148-153.

11. Zhengyan $Y$, Keke $L$, et al. Cone-beam computed tomography study of the root and canal morphology of mandibular permanent anterior teeth in a Chongqing population. Ther Clin Risk Manag 2016;12: $19-25$.

12. Soleymani A, Namaryan N, et al. Root Canal Morphology of Mandibular Canine in an Iranian Population: A CBCT Assessment. Iran Endod J 2017;12(1):78-82. DOI: 10.22037/iej.2017.16.

13. Green D. Double canals in single roots. Oral Surg Oral Med Oral Pathol 1973 May;35(5):689-696. DOI: 10.1016/0030-4220(73)90037-6.

14. Yousef $A D$, Abdullah $A$, et al. Cone-beam computed tomographic evaluation of root canal morphology of mandibular canines in a Saudi subpopulation. Saudi Endod J 2019;9:113-118.

15. Rahmatulla $\mathrm{M}, \mathrm{Wyne} \mathrm{AH}$. Bifid roots in a mandibular canine: Report of an unusual case. Saudi Dent J 1993;5:77-78.

16. Balto HA, Al-Wakeel M. Mandibular canine with two root canals-case report. Egypt Dent J 2007;53:2535-2538.

17. Alenezi MA. Permanent mandibular canine with two roots and two root canals: Two case reports. Saudi Endod J 2016;6:98-100.
18. Al Dahman Yousef $\mathrm{H}, \mathrm{Al}$ Hawwas Abdullah Y. A Root Canal Retreatment of Permanent Mandibular Canine with Two Canals - A Case Report. Adv Dent \& Oral Health 2017;4:4-7.

19. Sahoo HS, Amalavathy RK, et al. A Case Report on Endodontic Management of a Rare Vertucci Type III Maxillary Canine. 2019; 2019:4154067. DOI: 10.1155/2019/4154067.

20. Pan JYY, Parolia A, et al. Root canal morphology of permanent teeth in a Malaysian subpopulation using cone-beam computed tomography. BMC Oral Health 2019 Jan;19(1):14. DOI: 10.1186/s12903-019-0710-z.

21. Patel S, Dawood A, et al. New dimensions in endodontic imaging: part 1. Conventional and alternative radiographic systems. Int Endod J 2009 Jun;42(6):447-462. DOI: 10.1111/j.1365-2591.2008.01530.x.

22. Neelakantan $P$, Subbarao C, et al. Comparative evaluation of modified canal staining and clearing technique, cone-beam computed tomography, peripheral quantitative computed tomography, spiral computed tomography, and plain and contrast medium-enhanced digital radiography in studying root c. J Endod 2010 Sep;36(9):15471551. DOI: 10.1016/j.joen.2010.05.008.

23. von Zuben M, Martins JNR, et al. Worldwide Prevalence of Mandibular Second Molar C-Shaped Morphologies Evaluated by Cone-Beam Computed Tomography. J Endod 2017;43(9):1442-1447. DOI: 10.1016/ j.joen.2017.04.016.

24. Caliskan MK, Pehlivan Y, et al. Root canal morphology of human permanent teeth in a Turkish population. J Endod 1995 Apr;21(4):200204. DOI: 10.1016/S0099-2399(06)80566-2.

25. Miyoshi S, Fujiwara J, et al. Bifurcated root canals and crown diameter. J Dent Res 1977 Nov;56(11):1425. DOI: 10.1177/00220345770560112901.

26. Bellizzi R, Hartwell G. Clinical investigation of in vivo endodontically treated mandibular anterior teeth. J Endod 1983 Jun;9(6):246-248. DOI: 10.1016/S0099-2399(86)80022-X.

27. Kaffe I, Kaufman A, et al. Radiographic study of the root canal system of mandibular anterior teeth. Int Endod J 1985 Oct;18(4):253-259. DOI: 10.1111/j.1365-2591.1985.tb00452.x.

28. Sert S, Bayirli GS. Evaluation of the root canal configurations of the mandibular and maxillary permanent teeth by gender in the Turkish population. J Endod 2004 Jun;30(6):391-398. DOI: 10.1097/00004770200406000-00004.

29. Bakianian Vaziri P, Kasraee S, et al. Root Canal Configuration of Onerooted Mandibular Canine in an Iranian Population: An In Vitro Study. J Dent Res Dent Clin Dent Prospects 2008;2(1):28-32.

30. Rahimi S, Milani AS, et al. Prevalence of two root canals in human mandibular anterior teeth in an Iranian population. Indian J Dent Res 2013;24(2):234-236. DOI: 10.4103/0970-9290.116694.

31. Somalinga Amardeep N, Raghu S, et al. Root canal morphology of permanent maxillary and mandibular canines in Indian population using cone beam computed tomography. Anat Res Int 2014;2014:731859. DOI: 10.1155/2014/731859.

32. Martins JNR, Marques D, et al. Gender influence on the number of roots and root canal system configuration in human permanent teeth of a Portuguese subpopulation. Quintessence Int 2018;49:103-111. DOI: 10.3290/j.qi.a39508.

33. Martins JNR, Marques D, et al. Prevalence Studies on Root Canal Anatomy Using Cone-beam Computed Tomographic Imaging: A Systematic Review. J Endod 2019;45(4):372-386. 